\title{
6. TREFFEN DES ARBEITSKREISES ÖSTERREICHISCHER MEDI- ZINBIBLIOTHEKARINNEN UND -BIBLIOTHEKARE (WIEN, 13. JUNI 2016)
}

\author{
von Bruno Bauer
}

Am 13. Juni 2016 fand an der Universitätsbibliothek der Medizinischen Universität Wien das 6. Treffen des Arbeitskreises österreichischer Medizinbibliothekarinnen und -bibliothekare statt. Mit dieser Veranstaltung wurde an die erfolgreiche Tradition der Treffen in den Jahren 2005 bis 2007 angeknüpft. ${ }^{2}{ }^{4}$ Der Arbeitskreis österreichischer Medizinbibliothekarinnen und -bibliothekare ist einer von vier Arbeitskreisen, die im Rahmen der Arbeitsgemeinschaft für Medizinisches Bibliothekswesen (AGMB) eingerichtet worden sind. Die AGMB ist ein Verein zur Förderung des medizinischen Bibliotheks- und Informationswesens und weist ca. 450 Mitglieder in Deutschland, Österreich und der Schweiz auf. ${ }^{5}$

In einer Eröffnungsrunde berichteten die Teilnehmerinnen und Teilnehmer zunächst über die aktuelle Situation an ihren Einrichtungen und schilderten ihre Eindrücke von Veranstaltungen, die sie in jüngster Zeit besucht hatten.

Den Schwerpunkt des Treffens bildeten kurze Vorträge zu Themen aus den im Arbeitskreis vertretenen Bibliotheken.

Susanne Casagranda und Günter Androsch berichteten über die Herausforderung, eine optimale Literaturversorgung für die Angehörigen der 2014 an der Johannes Kepler Universität (JKU) Linz neu errichteten Medizinischen Fakultät von Grund auf zu organisieren. Gemäß den BolognaKriterien ist das Medizinstudium in ein Bachelor- und ein Masterstudium gegliedert. Eine Besonderheit der neuen medizinischen Ausbildungsstätte ist die Kooperation mit der Medizinischen Universität Graz, an der die vorklinische Ausbildung der Studierenden der Medizinischen Fakultät der Universität Linz stattfindet. Aus dieser engen Zusammenarbeit heraus entstand auch die Vorgabe, ein im Vergleich zur Medizinischen Universität Graz identes Angebot medizinischer e-Journals in Linz zu entwickeln. Diesbezüglich finden derzeit intensive Lizenzverhandlungen der Universitätsbibliothek Linz mit den großen Fachverlagen statt. ${ }^{6}$

Karin Cepicka informierte über die Erfahrungen der Universitätsbibliothek der Medizinischen Universität Wien seit dem Beginn des Zertifizierungsprozesses gemäß ISO 9001 im Jahr 2012. Die von der Norm geforderten Instrumente des Qualitätsmanagements sind mittlerweile im Bibli- 
otheksbetrieb sehr gut verankert. Ein wesentlicher Vorteil liegt in der systematischen Behandlung von Kritik und Verbesserungsvorschlägen. Das im ersten Halbjahr 2016 durchgeführte Überwachungsaudit wurde auf Basis der aktualisierten Norm ISO 9001:2015 vorgenommen. Damit einhergehend sind auch Themen wie Risikomanagement, Change Management und Wissensmanagement in den Fokus des Zertifizierungsverfahrens gerückt.

Daran anschließend erfolgte ein Erfahrungsaustausch über den Einsatz von elektronischen Büchern an Universitätsbibliotheken. Daniel Formanek berichtete darüber, dass der diesbezügliche Schwerpunkt an der Universitätsbibliothek der Medizinischen Universität Wien aufTiteln liegt, die für das Medizinstudium von Bedeutung sind. So ist etwa die Hälfte der ca. 300 Titeln der Lehrbuchsammlung auch in elektronischer Form verfügbar. Bemerkenswert ist in diesem Zusammenhang die Tatsache, dass die mittlerweile enorm angestiegene Nutzung der elektronischen Bücher keinen unmittelbaren Rückgang bei den Entlehnungen bewirkt hat. In einer im Vorjahr durchgeführten BenutzerInnen-Befragung wurde von zirka einem Drittel der Studierenden angemerkt, dass sie die e-Versionen der Lehrbücher präferieren, während ein weiteres Drittel die Print-Versionen bevorzugt. Das dritte Drittel hat keine Präferenz für eine bestimmte Medienform und nutzt je nach konkretem Anlassfall die Print- oder die Online-Version.

Über die Beteiligung der Universitätsbibliothek am neuen Curriculum der Veterinärmedizinischen Universität Wien informierten David Frank und Claudia Hausberger in ihrer Präsentation. Ziele des neuen Curriculums sind die Vermittlung von theoretischem und praktischem Wissen, das Einüben und Fördern von psychosozialen Fähigkeiten, die Vermittlung von praktischen Fertigkeiten sowie die Formung von ethischen Grundhaltungen. In der Lehrveranstaltung „Wissenschaft in der Veterinärmedizin“, einem Lernmodul des ersten Semesters, werden das Verfassen einer wissenschaftlichen Arbeit, Statistik und Wissenschaftstheorie vermittelt. Inhalte, die von der Universitätsbibliothek eingebracht werden, sind Recherche und deren Dokumentation, Literaturverwaltung, Zitierregeln der Universität sowie Urheberrecht. In Gruppen zu 40 Studierenden werden jeweils Vorlesung und Übung angeboten, wobei im Rahmen der Übungen konkrete Aufgabenstellungen in einem EDV-Raum bearbeitet werden. Für die Universitätsbibliothek ist die Beteiligung an der Lehrveranstaltung eine gute Möglichkeit, allen Studierenden in einem frühen Stadium ihres Studiums die Bedeutung der Bibliothek und deren Angebote vermitteln zu können.

Helmut Dollfuß bot einen Einblick in innovative Projekte, die an der Universitätsbibliothek der Medizinischen Universität Wien in jüngster Zeit betrieben werden. Zunächst sprach er über die Erfahrungen mit dem Ein- 
satz des EZproxy, der sich an der Medizinischen Universität Wien als kostengünstige Möglichkeit für die Organisation eines authentifizierten Zugriffs auf die elektronischen Inhalte der eigenen Institution an der Medizinischen Universität Wien bestens bewährt hat. ${ }^{7}$ Anschließend erläuterte er, welche Bibliotheksanwendungen für mobile Endgeräte es im medizinischen Bereich bereits gibt. Zu nennen sind in diesem Zusammenhang optimierte Webseiten für mobile Endgeräte sowie Apps, die herunter geladen werden können. Danach stellte er UpToDate und Dynamed vor und sprach über die aktuellen Verbesserungen und Erweiterungen dieser klinischen Informationstools, die an der Medizinischen Universität Wien zum Einsatz kommen.

Das Institutional Repository der Vinzenz-Gruppe wurde von Christian Vogel vorgestellt. Diese zentrale institutionelle Veröffentlichungsdatenbank wurde auf Basis der Software EPrints aufgebaut und steht für Publikationen aller Mitarbeiterinnen und Mitarbeiter der Häuser der VinzenzGruppe offen, wobei Open Access-Qualitätsstandards eingehalten werden. ${ }^{8}$

Über nationale Kooperationsprojekte in Österreich informierte Bruno Bauer. Es gibt zwar viele Themen, die gemeinschaftlich ausschließlich von von Medizinbibliothekarinnen und Medizinbibliothekaren bearbeitet werden können, viele Herausforderungen betreffen allerdings alle wissenschaftlichen Bibliotheken, unabhängig von ihrer fachlichen Schwerpunktsetzung.

Besonders erfolgreich erweist sich die Kooperation bei e-Infrastructures Austria, einem vom BMWFW geförderten Hochschulraumstrukturmittelprojekt (2014-2016), bei dem 25 Einrichtungen in den Themenfeldern Repositorien und Forschungsdaten zusammenarbeiten. ${ }^{9}$

Ähnlich innovativ war der Abschluss des aktuellen Lizenzvertrages für die Zeitschriften des Springer-Verlages, der über die Kooperation E-Medien Österreich (KEMÖ) realisiert werden konnte und der auch international große Beachtung gefunden hat. Der neue Springer Compact-Vertrag (Laufzeit 2016-2018) bietet den 34 beteiligten Einrichtungen neben einer Access-Komponente, die den Kooperationspartnern Zugriff auf alle Springer-Zeitschriften einräumt, auch eine Open Access-Komponente, wodurch alle Angehörigen der kooperierenden Einrichtungen als Autorinnen und Autoren ihre Fachbeiträge bei den Springer-Zeitschriften ohne zusätzliche Kosten Open Access publizieren können. ${ }^{10}$

Als eigener Programmpunkt auf der Agenda stand die aktuelle Entwicklung an der Deutschen Zentralbibliothek für Medizin. Aufgrund eines Beschlusses des Senates der Leibniz Gemeinschaft vom 17. März 2016 wurde die Beendigung der Förderung von ZB MED - Leibniz-Informationszentrum Lebenswissenschaften in Köln empfohlen. ${ }^{11}$ 
Von allen Beteiligten wurde große Betroffenheit hinsichtlich dieser unverständlichen Entscheidung bekundet, verbunden mit der Hoffnung, dass eine gute Lösung gefunden wird, damit die hervorragenden Services dieser über Jahrzehnte in der medizinischen Literaturversorgung bestens etablierten Einrichtung auch weiterhin genutzt werden können. Die drohende Abwicklung ist umso bedauerlicher, als erst zum Jahreswechsel 2015/2016 angekündigt worden war, dass die ZB MED einen Teil der Datenbanken des DIMDI übernehmen wird, weil dieser Bereich dort ab 2016 nicht mehr weiterbetrieben werden soll. ${ }^{12}$

Hingewiesen wurde auch auf die Online-Petition von Rudolf Mumenthaler zum Erhalt der ZB MED, die von knapp 10.000 Unterstützerinnen und Unterstützern unterzeichnet worden ist; diese wurde mittlerweile an die Gemeinsame Wissenschaftskonferenz übergeben. ${ }^{13}$

Von den Mitgliedern des Arbeitskreises österreichischer Medizinbibliothekarinnen und -bibliothekare wurde übereinstimmend der Hoffnung Ausdruck verliehen, dass eine zukunftsweisende Lösung für den Fortbestand der ZB MED gefunden wird.

Zum Abschluss des 6. Treffens des Arbeitskreises österreichischer Medizinbibliothekarinnen und -bibliothekare sprachen Claudia Hausberger und David Frank eine herzliche Einladung zur Teilnahme an der Jahrestagung der Arbeitsgemeinschaft für Medizinisches Bibliothekswesen im nächsten Jahr aus, die von 25. bis 27. September 2017 an der Veterinärmedizinischen Universität Wien stattfinden wird.

Mag. Bruno Bauer

Vorsitzender des Arbeitskreises österreichischer Medizinbibliothekarinnen und -bibliothekare Universitätsbibliothek der Medizinischen Universität Wien Währinger Gürtel 18-20, A-1097 Wien E-Mail: bruno.bauer@meduniwien.ac.at

1 Bauer, Bruno: Bericht über das 1. Treffen österreichischer Medizinbibliothekarinnen und -bibliothekare. - In: Mitteilungen der Vereinigung Österreichischer Bibliothekarinnen und Bibliothekare 58 (2005), H. 2, S. 88-95.

2 Bauer, Bruno: Bericht über das 2. Treffen österreichischer Medizinbibliothekarinnen und -bibliothekare. - In: Mitteilungen der Vereinigung Österreichischer Bibliothekarinnen und Bibliothekare 58 (2005), H. 4, S. 84-88. 
3 Bauer, Bruno: Bericht über das 3. Treffen österreichischer Medizinbibliothekarinnen und -bibliothekare am 22. Juni 2006 in der Gesellschaft der Ärzte. - In: Mitteilungen der Vereinigung Österreichischer Bibliothekarinnen und Bibliothekare 59 (2006), H. 2, S. 69-74.

4 Bauer, Bruno: Bericht über das 4. Treffen österreichischer Medizinbibliothekarinnen und -bibliothekare am 4. Dezember 2006 am Ludwig Boltzmann Institut für Health Technology Assessment. - In: Mitteilungen der Vereinigung Österreichischer Bibliothekarinnen und Bibliothekare 60 (2007), H. 1, S. 67-71.

5 Arbeitsgemeinschaft für Medizinisches Bibliothekswesen (AGMB) / Website. Online unter: http://www.agmb.de/.

6 Johannes Kepler Universität Linz: Medizinische Fakultät. Online unter: http://www.jku.at/content/e213/e236890.

7 OCLC: EZproxy. Online unter: https://www.oclc.org/de-DE/ezproxy. html.

8 Vinzenz Gruppe: Veröffentlichungsdatenbank/Institutional Repository. Online unter: http://eprints.vinzenzgruppe.at/.

9 e-Infrastrucutres Austria / Website. Online unter: https://www.e-infrastructures.at/.

10 Kooperation E-Medien Österreich (KEMÖ): Infoseite zum Open-Access-Publizieren mit Springer Compact. Online unter: https://www. konsortien.at/springercompact.asp.

11 Senat der Leibniz-Gemeinschaft: Stellungnahme zur Deutschen Zentralbibliothek für Medizin - Leibniz-Informationszentrum Lebenswissenschaften, Köln / Bonn (ZB MED). Online unter: http://www. leibniz-gemeinschaft.de/fileadmin/user_upload/downloads/Evaluierung/Senatsstellungnahmen/ZB_MED_-_Senatsstellungnahme_ vom_17_03_2016_mit_Anlagen.pdf.

12 Evidenz in der Medizin: Medizinische Literaturdatenbanken in Deutschland vor dem Aus. - In: DAZ online. Online unter: https://www.deutsche-apotheker-zeitung.de/news/artikel/2016/03/24/medizinischeliteraturdatenbanken-in-deutschland-vor-dem-aus.

13 Rudolf Mumenthaler: Keep ZB MED - gegen die Schliessung von ZB MED. Offene Petition zur Erhaltung von ZB MED als überregionale Forschungsinfrastruktureinrichtung. Online unter: https://www.change. org/p/keepzbmed.

Dieses Werk ist lizenziert unter einer

Creative-Commons-Lizenz Namensnennung 4.0 International 